\title{
Myocardial blood flow assessment with SPECT systems: The renovation continues
}

\author{
Gil Kovalski, PhD, ${ }^{\mathrm{a}}$ and Tali Sharir, $\mathrm{MD}^{\mathrm{b}}$ \\ a General Electric Healthcare, Haifa, Israel \\ b Department of Nuclear Cardiology, Assuta Medical Centers, Tel Aviv, Israel, and Faculty of \\ Health Sciences, Ben-Gurion University of the Negev, Beersheba, Israel
}

Received Feb 14, 2019; accepted Feb 14, 2019

doi: $10.1007 / \mathrm{s} 12350-019-01666-3$

See related article, pp. 2287-2302

\section{THE SCOPE}

The incremental prognostic value of myocardial blood flow (MBF) and coronary flow reserve (CFR) by $\mathrm{PET} / \mathrm{CT}$ has been well demonstrated in several studies. ${ }^{1,2}$ It was also shown that MBF can be obtained with CZT-based SPECT systems having dynamic imaging capability. ${ }^{3-10}$ Recently, several investigators demonstrated the feasibility of quantifying MBF with NaIbased dual-head SPECT systems equipped with rapid rotational gantry (RRG). ${ }^{11}$ In this edition of the journal of nuclear cardiology, Rongzheng et al. from Fu Wai Hospital, China, performed a head to head comparison between resting MBF obtained by a conventional SPECT system with RRG and a CZT-SPECT system in 20 patients with congestive heart failure (CHF subgroup) and left ventricular systolic dysfunction (ejection fraction $<50 \%$ by echocardiography), and 20 non-CHF patient group with normal systolic function. The majority of the CHF patients (19 of 20) were diagnosed as suffering from ischemic heart disease. All patients (20 CHF and 20 non-CHF) were scanned on both dual-head NaI-based SPECT system and a CZTSPECT system on two consecutive days. The results demonstrated good correlation between resting MBF by conventional and CZT-SPECT systems in CHF and nonCHF patients, with good repeatability. Moreover, resting

\footnotetext{
Reprint requests: Gil Kovalski, PhD, General Electric Healthcare, Haifa, Israel; gil.kovalski@ge.com

J Nucl Cardiol 2020;27:2303-5.

$1071-3581 / \$ 34.00$

Copyright (C) 2019 American Society of Nuclear Cardiology.
}

MBF was lower among CHF patients compared to nonCHF patients for both SPECT systems.

\section{WHAT IS THE CLINICAL VALUE OF MBF?}

It was well demonstrated that myocardial perfusion imaging (MPI) provides a valuable diagnostic and prognostic information. However, myocardial perfusion is displayed as a relative distribution of the radiotracer uptake in the myocardium where the highest count density voxel is presented with the brightest color in the selected color scheme. Therefore, the absolute tissue uptake remains unknown which may result in a normal distribution pattern despite a global reduction of the radiotracer uptake. MBF provides an absolute quantification of the myocardial tissue uptake by converting the count density values into absolute flow unit $(\mathrm{mL} / \mathrm{min} / \mathrm{g})$. Therefore, a global reduction of radiotracer count density which may have a normal perfusion pattern will be reflected by low flow values. As demonstrated in several studies, the diagnostic power is increased when flow measurements are included ${ }^{1,2,12,13}$ which may also increase the adoption of stress-only protocols, lower radiation burden, and cost saving.

It should also be noted that SPECT and SPECT/CT systems are widely available globally therefore the infrastructure of adopting that technique is in place.

\section{TECHNICAL REQUIREMENTS FOR MBF CALCULATION}

In a nutshell, MBF calculation requires capturing the radiotracer transit through the left ventricle which is often referred as the arterial "input function" component of the kinetic model. This component is often obtained by a dynamic acquisition initiated prior to radiotracer injection and typically lasts for 8 to 10 minutes. The second component is the radiotracer 
perfusion uptake in the myocardium which is measured either by increasing the dynamic acquisition time duration or obtained as a separate perfusion acquisition. ${ }^{3,5,6}$ These two components are then used to assess the compartment modeling constants $\mathrm{K} 1$ and optionally k2 followed by conversion into flow units. High sensitivity systems are suited for blood flow quantification due to the fact that they allow reliable input function assessment. In addition, suitable kinetic modeling and radiotracers with high extraction characteristics shall be used to avoid underestimation at high flow rates. ${ }^{14}$ These requirements are in principle been addressed in PET imaging having high sensitivity systems and high extraction radiotracers such as ${ }^{13} \mathrm{~N}$-ammonia and ${ }^{15} \mathrm{O}$ water. ${ }^{1,12,13}$ However, a delicate process is needed to obtain MBF in a robust manner and for the appropriate clinical indication. ${ }^{15,16}$ The later statement is obviously relevant for SPECT techniques which currently have lower system sensitivity and radiotracer kinetics (of ${ }^{99 \mathrm{~m}} \mathrm{TC}$ ) as compared to PET. In recent years, revolutionized CZT-based cardiac-dedicated SPECT systems having 5- to 8-fold increase in system sensitivity while maintaining spatial resolution as compared to dual-head systems were introduced. ${ }^{17,18}$ These systems have a dynamic capability to capture the radiotracer transit through the left ventricle. Several studies have shown that MBF quantification can be obtained with these systems. $^{3-9,19}$

Along with the efforts to optimize the protocols and performance to calculate MBF using CZT-based systems, investigators are seeking for techniques to obtain MBF using conventional dual-head rotating SPECT and SPECT/CT systems. ${ }^{11}$ The main challenge for these systems is to obtain the input function in a robust manner despite the inconsistency in the projection images due to the rotation of the system heads during the dynamic transit of the radiotracer through the left ventricle. The input function can be obtained using dynamic stationary planar image $;^{20}$ however, this technique is limited by the two-dimensional nature of the data and therefore was not widely adopted in clinical practice.

\section{ARE WE READY FOR PRIME TIME?}

The majority of nuclear cardiac studies are performed to date using SPECT systems, and most of these use a conventional rather than a CZT system. Several editorials outlined the potential of SPECT to follow PET and provide flow assessment in clinical routine. ${ }^{21,22}$ In this edition of the journal of nuclear cardiology, Rongzheng et al. compared the resting MBF of CHF and non-CHF patients using a cardiac stationary CZT system and a rotating dual-head NaI system. The system heads of the dual-head rotating camera were rotated back-and-forth in order to obtain the arterial input function and radiotracer transition through the left ventricle at a maximal speed of 10 seconds per rotation. Angular inconsistencies were corrected by back-projection and forward projection technique of the empty angles. The authors also applied tissue attenuation, scatter, resolution recovery, spill over, and partial volume corrections during reconstruction prior to $\mathrm{MBF}$ quantification. High resting MBF correlation between the CZT and the dual-head system for both populations (CHF and non-CHF) was reported. As noted by the authors, a major limitation of the study is the fact that MBF was assessed in resting mode only. Additionally, no gold standard was available in this study. The primary technical ingredients required for myocardial blood flow assessment by SPECT are outlined which increase the importance of the manuscript as an educational resource for quantifying $\mathrm{MBF}$ using SPECT systems.

Additional test-retest studies are in the pipeline which will further explore the domain of MBF quantification with high sensitivity SPECT systems. It is expected that these studies will formulize the optimal protocols for MBF assessment with SPECT. However, to echo Juneau et $\mathrm{al}^{15}$ let us ensure we walk before we fly in SPECT as well as in PET.

\section{Disclosure}

Gil Kovalski is an employee of GE Healthcare. Tali Sharir has nothing to disclose.

\section{References}

1. Murthy VL, Naya M, Foster CR, Hainer J, Gaber M, Di Carli G, Blankstein R, Dorbala S, Sitek A, Pencina MJ, Di Carli MF. Improved cardiac risk assessment with noninvasive measures of coronary flow reserve. Circulation. 2011;124:2215-24.

2. Murthy VL, Bateman TM, Beanlands RS, Berman DS, BorgesNeto S, Chareonthaitawee P, Cerqueira MD, DePuey EG, Dilsizian V, Dorbala S, Ficaro EP. Clinical quantification of myocardial blood flow using PET: Joint position paper of the SNMMI cardiovascular council and the ASNC. J Nucl Med 2018;25(1):269-97.

3. Wells RG, Timmins R, Klein R, Lockwood J, Marvin B, Wei L, Ruddy TD. Dynamic SPECT measurement of absolute myocardial blood flow in a porcine model. J Nucl Med 2014;55(10):1685-91.

4. Agostini D, Roule V, Nganoa C, Roth N, Baavour R, Parienti JJ, Beygui F, Manrique A. First validation of myocardial flow reserve assessed by dynamic 99mTc-sestamibi CZT-SPECT camera: head to head comparison with 15O-water PET and fractional flow reserve in patients with suspected coronary artery disease. The WATERDAY study. Eur J Nucl Med 2018;45(7):1079-90.

5. Han S, Kim YH, Ahn JM, Kang SJ, Oh JS, Shin E, Sung C, Chae SY, Park SJ, Grimberg G, Kovalski G, Moon DH. Feasibility of dynamic stress $201 \mathrm{Tl} /$ rest $99 \mathrm{mTc}$-tetrofosmin single photon 
emission computed tomography for quantification of myocardial perfusion reserve in patients with stable coronary artery disease. Eur J Nucl Med Mol Imaging. 2018;45(12):2173-80.

6. Ben Bouallègue F, Roubille F, Lattuca $\mathrm{B}$, Cung TT, Macia JC, Gervasoni R, Leclercq F, Mariano-Goulart D. SPECT myocardial perfusion reserve in patients with multi-vessel coronary disease: Correlation with angiographic findings and invasive fractional flow reserve measurements. J Nucl Med 2015;56(11):1712-7.

7. Shiraishi S, Sakamoto F, Tsuda N, Yoshida M, Tomiguchi S, Utsunomiya D, Ogawa H, Yamashita Y. Prediction of left main or 3-vessel disease using myocardial perfusion reserve on dynamic thallium-201 single-photon emission computed tomography with a semiconductor gamma camera. Circ J 2015;79(3):623-31.

8. Nkoulou R, Fuchs TA, Pazhenkottil AP, Kuest SM, Ghadri JR, Stehli J, Fiechter M, Herzog BA, Gaemperli O, Buechel RR, Kaufmann PA. Absolute myocardial blood flow and flow reserve assessed by gated SPECT with cadmium-zinc-telluride detectors using 99mTc-tetrofosmin: head to head comparison with $13 \mathrm{~N}$ ammonia PET. J Nucl Med 2016;57:1887-92.

9. Wells RG, Marvin B, Poirier M, Renaud J, Ruddy TD. Optimization of SPECT measurement of myocardial blood flow with corrections for attenuation, motion, and blood binding compared with PET. J Nucl Med 2017;58(12):2013-9.

10. Ben-Haim S, Murthy VL, Breault C, Allie R, Sitek A, Roth N, Fantony J, Moore SC, Park MA, Kijewski M, Haroon A, Slomka P, Erlandsson K, Baavour R, Zilberstien Y, Bomanji J, Di Carli MF. Quantification of myocardial perfusion reserve using dynamic SPECT imaging in humans: a feasibility study. J Nucl Med 2013;54:873-9.

11. Hsu B, Hu LH, Yang BH, Chen LC, Chen YK, Ting CH, Hung GU, Huang WS, Wu TC. SPECT myocardial blood flow quantitation toward clinical use: a comparative study with $13 \mathrm{~N}$ Ammonia PET myocardial blood flow quantitation. Eur $\mathbf{J}$ Nucl Med Mol Imaging 2017;44(1):117-28.

12. Di Carli MF. Measurement of MBF by PET is ready for prime time as an integral part of clinical reports in diagnosis and risk assessment of patients with known or suspected CAD-PRO. J Nucl. Cardiol. 2017. https://doi.org/10.1007/s12350-017-1035-4.

13. Parkash R, deKemp RA, Ruddy TD, Kitsikis A, Hart R, Beauchesne L, Beauschene L, Williams K, Davies RA, Labinaz
M, Beanlands RSB. Potential utility of rubidium 82 PET quantification in patients with 3-vessel coronary artery disease. J Nucl Cardiol 2004;11:440-9.

14. Liu Chi, Sinusas Albert. Is assessment of absolute myocardial perfusion with SPECT ready for prime time? J Nucl Med 2014;55(10):1573-5.

15. Juneau D, Beanlands RS. Reporting myocardial flow reserve with PET Ready or not, here it is But walk before you fly. J Nucl Cardiol 2017;25:164-8.

16. Schelbert H. Measurement of MBF by PET is ready for prime time as an integral part of clinical reports in diagnosis and risk assessment of patients with known or suspected CAD For prime time not yet-need impact and certainty. J Nucl Cardiol 2018;25(1):153-6.

17. Sharir T, Ben-Haim S, Merzon K, Prochorov V, Dickman D, Berman DS. High-speed myocardial perfusion imaging initial clinical comparison with conventional dual detector anger camera imaging. JACC Cardiovasc. Imaging. 2008;1:156-63.

18. Esteves FP, Raggi P, Folks RD, Keidar Z, Askew JW, Rispler S, et al. Novel solid-state-detector dedicated cardiac camera for fast myocardial perfusion imaging: multicenter comparison with standard dual detector cameras. J Nucl Cardiol 2009;16:927-34.

19. Lee JS, Kovalski G, Sharir T, Lee DS. Advances in imaging instrumentation for nuclear cardiology. J Nucl Cardiol 2017. h ttps://doi.org/10.1007/s12350-017-0979-8.

20. Sugihara H, Yonekura Y, Kataoka K, Fukai D, Kitamura N, Taniguchi Y. Estimation of coronary flow reserve with the use of dynamic planar and SPECT images of Tc-99m tetrofosmin. J Nucl Cardiol 2001;8:575-9.

21. Slomka PJ, Berman DS, Germano G. Absolute myocardial blood flow quantification with SPECT/CT: Is it possible? Nucl. Cardiol. 2014;21:1092-5.

22. Ernest V. Garcia Are SPECT measurements of myocardial blood flow and flow reserve ready for clinical use? Eur J Nucl Med Mol Imaging 2014;41(12):2291-3.

Publisher's Note Springer Nature remains neutral with regard to jurisdictional claims in published maps and institutional affiliations. 